\title{
Exploration of Novel Nano-scale Instabilities in Metastable Beta Titanium Alloys Using Transmission Electron Microscopy and Aberration-Corrected Scanning Transmission Electron Microscopy
}

\author{
Yufeng Zheng ${ }^{1}$, Rajarshi Banerjee ${ }^{2}$ and Hamish L. Fraser ${ }^{1}$ \\ 1. Center for the Accelerated Maturation of Materials and Department of Materials Science and \\ Engineering, The Ohio State University, Columbus, OH, USA. \\ 2. Department of Materials Science and Engineering, University of North Texas, Denton TX, USA
}

Recently, metastable beta titanium alloys have attracted considerable attention in the field of aerospace and bio-medical industries, due to the combination of attractive properties, such as high strength, low modulus, corrosion resistance and bio-compatibility. Compared with the most widely used alpha/beta titanium alloy, Ti-6Al-4V (wt.\%), the microstructure in metastable beta titanium alloys can be manipulated by various thermo-mechanical processing routes that often involve the precipitation of fine scaled intragranular $h c p$ structure alpha phase, with tailored fineness of the distribution of the precipitate phase depending on the given heat-treatment, generated by the influence of nano-scale structural and compositional instabilities. With the technological improvement in transmission electron microscopy, especially the probe aberration corrected scanning transmission electron microscopy, it has enabled the atomic resolution Z-contrast high angle annular dark field- scanning transmission electron microscopy (HAADF-STEM) imaging, which is capable of characterizing the atomic column configurations with a sub-angstrom probe [1] and provides novel insights of nano-scale structures in titanium alloys. In our recent studies coupling aberration-corrected scanning transmission electron microscopy and 3D atom probe tomography, it has been shown that the nano-scale metastable hexagonal structure isothermal omega phase particles in the parent $b c c$ structure beta phase can significantly affect the size, morphology and number density of alpha precipitates in Ti-5Al-5Mo-5V-3Cr (Ti-5553, wt.\%) upon different heat treatments by providing additional driving force for super-refined alpha nucleation from the compositional and stress field associated with these nano-scale particles [2].

In the first part of this current work, a newly characterized nano-scale structural instability termed as $\mathrm{O}^{\prime}$ phase in the Ti-26Nb-2Zr (at.\%) beta type titanium alloy is to be introduced [3]. This new phase was investigated using conventional transmission electron microscope and probe corrected scanning transmission electron microscope (FEI Titan ${ }^{\mathrm{TM}}$ 80-300). A large number density of nano-scale particles is shown in the dark field image in Fig. 1(a), and the structure of $\mathrm{O}^{\prime}$ phase particles is shown in the HAADFSTEM image in Fig. 1(b), of which in the lower left part, marked by the dashed red box, the bcc symmetry has been broken by small degree of displacement of every other atomic column. These displacements result in the orthorhombic structure shown in the inset of Fig. 1(b), which describes the result of the $\{110\}<1 \overline{1} 0>$ shuffle $Z$ (soft phonon).

In the second part of this work, the crystallography and the structure of a previously unidentified ordered orthorhombic metastable phase, named O" phase, in the beta matrix of Ti-5553 were studied using conventional transmission electron microscope and probe corrected scanning transmission electron microscope (FEI Titan ${ }^{\mathrm{TM}}$ 80-300) [4]. The dark field image, shown in Fig. 2(a), shows the formation of ellipsoidal morphology nano-scale particles in Fig. 2(a) and the Z-contrast HAADF-STEM imaging reveals a novel ordering mechanism in beta titanium alloy for the first time that one in every three $\{110\}$ beta planes exhibits a relative lower intensity than the other two as shown in Fig 2(b). The ordered 
orthorhombic structure O" phase obeys the following OR with the parent $\beta$ matrix: $(001)_{O^{\prime \prime}} /(011)_{\beta}$ and $[100]_{\mathrm{O}} / /[100]_{\beta}$, with the lattice parameter $a=0.328 \mathrm{~nm}, b=0.464 \mathrm{~nm}$ and $c=1.393 \mathrm{~nm}$, shown in Fig 2(c) [4].

References:

[1] HL Fraser et al., in „Transmission Electron Microscopy for Physical Metallurgists Elsevier Science“, (Burlington).

[2] Y Zheng et al., Acta Materialia 103 (2016), p. 850.

[3] Y Zheng et al., Scripta Materialia 116 (2016), p. 49.

[4] Y Zheng et al., Scripta Materialia 113 (2016), p. 202.
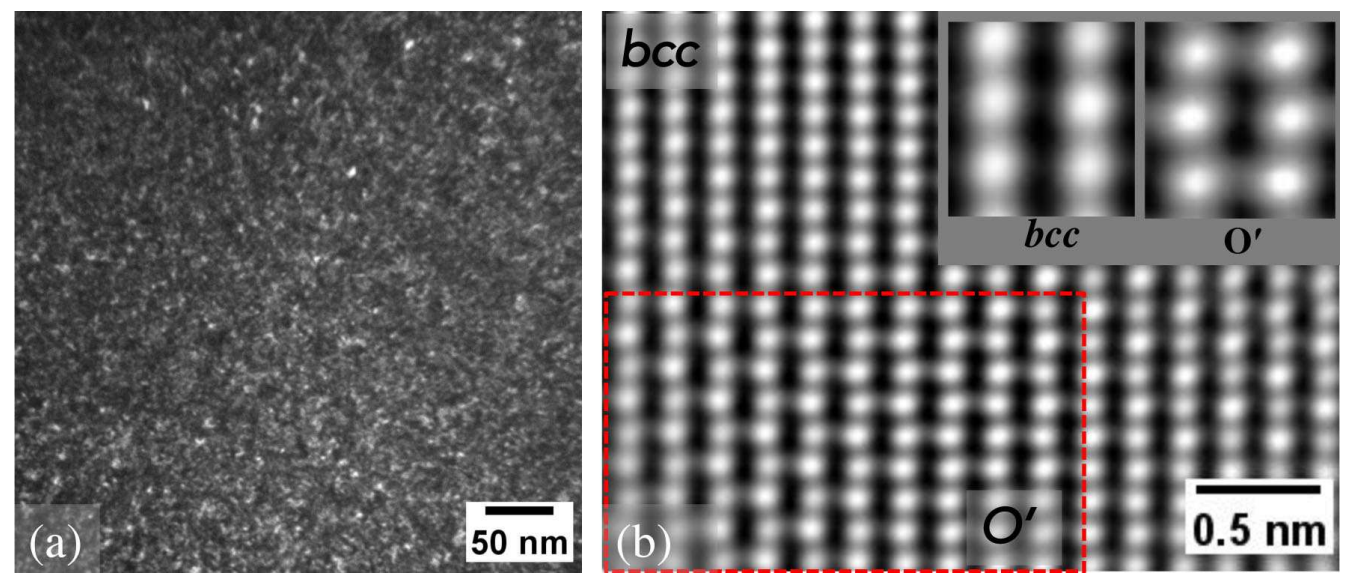

Figure 1. (a) Dark field image showing nano-scale particles; (b) Z-contrast HAAD-STEM image showing the nano-scale disordered orthorhombic structure O' phase [3].
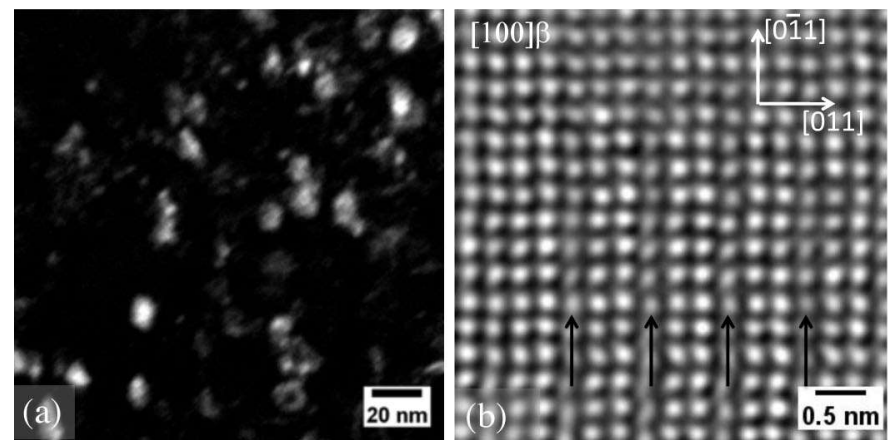
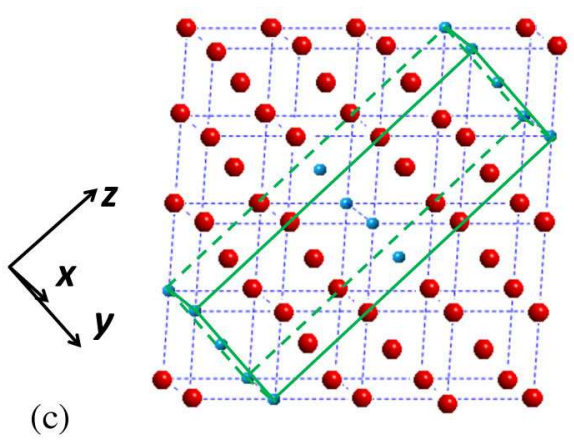

Figure 2. (a) Dark field image showing nano-scale O" particles; (b) Z-contrast HAAD-STEM image showing the nano-scale ordered orthorhombic structure $\mathrm{O}^{\prime \prime}$ phase; (c) schematic figure showing the structure of $\mathrm{O}^{\prime \prime}$ phase and its orientation relationship with beta phase matrix [4]. 